\title{
ESTUDO DE INCLUSÕES FLUIDAS EM VEIO DE QUARTZO AURÍFERO DO PROSPECTO PATINHAS, PROVÍNCIA AURÍFERA DO TAPAJÓS, CRÁTON AMAZÔNICO
}

\author{
EVANDRO LUIZ KLEIN*, LÚCIA TRAVASSOS DA ROSA-COSTA \& \\ JOSÉ MARIA DE AZEVEDO CARVALHO
}

\begin{abstract}
FLUID INCLUSION STUDY IN THE GOLD-BEARING QUARTZ VEIN AT THE PATINHAS PROSPECT, TAPAJÓS GOLD PROVINCE, AMAZONIAN CRATON The Patinhas prospect, in southeastern Tapajós province, consists of a gold-bearing quartz vein, averaging $70 \mathrm{~cm}$ in thickness, hosted by a subvertical, NE-SW-trending, brittle-ductile, strike-slip shear zone, cutting a granodioritic gneiss of the Cuiú-Cuiú complex $(2.0 \mathrm{Ga})$. The quartz is milky massive and sulfide-rich in its central portion, and foliated at the contact with the host rock. This contact is also outlined by a narrow hydrothermal alteration halo, containing sulfide dissemination, and by the ductile deformation superimposed to the host rock during shearing. The structural evidence suggests emplacement of the vein at a moderate depth, in an active structure, whose activity outlasted the vein formation. Gold occurs in microscopic particles that fill microfractures in pyrite crystals. Under the microscope, large quartz crystals are surrounded by a mosaic of recrystallized small grains. Weak to strong effects of ductile deformation are observable. Fluid inclusions (FI) occur as intraand transgranular trails, in the boundaries of recrystallized grains, or in the cores of less deformed and/or preserved relic quartz, where they are isolated or form small clusters of randomly-distributed inclusions. FI containing $\mathrm{CO}_{2}$, mixed $\mathrm{CO}_{2}-\mathrm{H}_{2} \mathrm{O}$, and $\mathrm{H}_{2} \mathrm{O}$ have been recognized. Among the aqueous inclusions two subgroups have been identified: a group of late FI, and a group of inclusions that are spatially associated with the $\mathrm{CO}_{2}$-bearing FI. The $\mathrm{CO}_{2}$ melting temperature of the $\mathrm{CO}_{2}$-bearing FI clustered close to the temperature of the triple point of the $\mathrm{CO}_{2}\left(-56.6^{\circ} \mathrm{C}\right)$. Based on structural, petrographic and microthermometric evidence, the fluid inclusions are interpreted as formed by a combination of phase separation and re-equilibration of an aqueous-carbonic fluid during and after their entrapment. Physico-chemical parameters estimated from the microthermometric results indicate that mineralization occurred between $307^{\circ}$ and $389^{\circ} \mathrm{C}$, at variable pressure (average of $2.1 \mathrm{~kb}$, corresponding to a depth of ca. $7 \mathrm{~km}$ ). The aqueous-carbonic fluid has bulk densities between 0.73 e $0.97 \mathrm{~g} / \mathrm{cm}^{3}$, mean salinity of $6.6 \mathrm{wt}$. \% NaCl equiv., and $\mathrm{XCO}_{2}$ of $6-16$ moles \%. These characteristics are compatible with those of structurally-controlled, mesothermal / mesozonal gold deposits.
\end{abstract}

Keywords: gold, metallogeny, fluid inclusions, $\mathrm{CO}_{2}$, Tapajós, Paleoproterozoic

Resumo O prospecto Patinhas, na porção sudeste da província Tapajós, é caracterizado por um veio de quartzo aurífero com espessura média de $70 \mathrm{~cm}$ hospedado em zona de cisalhamento rúptil-dúctil, transcorrente, subvertical, orientada segundo NE-SW, que corta gnaisse granodiorítico do Complexo Cuiú-Cuiú $(\sim 2,0 \mathrm{Ga})$. O quartzo é leitoso, maciço e rico em pirita em sua porção central e foliado no contato com a rocha encaixante. Esse contato é também marcado por estreito halo de alteração hidrotermal que contém sulfetos disseminados e pela deformação dúctil imposta à rocha encaixante pelo cisalhamento. $\mathrm{O}$ caráter estrutural é sugestivo de posicionamento do veio em profundidade moderada, em estrutura ativa, cuja atividade estendeu-se por algum tempo após a formação do veio. O ouro é microscópico e preenche microfraturas em cristais de pirita. Ao microscópio, cristais de quartzo de dimensões variadas são envoltos por matriz recristalizada, em mosaico. São observados efeitos variáveis de deformação dúctil, fraca a intensa, devido à deformação que acompanhou e sucedeu a formação do veio de quartzo. Inclusões fluidas (IF) ocorrem em trilhas intra- e transgranulares, em bordas de cristais recristalizados, ou são encontradas em áreas menos deformadas e/ou reliquiares do quartzo, de forma isolada ou em pequenos grupamentos aleatórios. São descritas inclusões carbônicas (tipo 1) mono- e bifásicas, geralmente associadas com inclusões aquo-carbônicas (tipo 2) bi- ou trifásicas em temperatura ambiente, com o volume de $\mathrm{CO}_{2}$ variando entre 20 e 70\%. Inclusões aquosas (tipo 3) bifásicas dividem-se em dois subtipos, um associado espacialmente aos tipos 1 e 2 e um outro posterior a todos os demais tipos. A temperatura de fusão da fase carbônica das IF dos tipos 1 e 2 mostra forte concentração de valores próximo ao ponto de fusão do $\mathrm{CO}_{2}$ puro. Com base no conjunto de dados estruturais, petrográficos e microtermométricos, interpretase a origem das IF como uma combinação entre separação de fases e re-equilibrio durante e após o aprisionamento. Parâmetros físicoquímicos estimados a partir dos dados microtermométricos indicam que: a mineralização ocorreu entre $307^{\circ} \mathrm{C}$ e $389^{\circ} \mathrm{C}$, sob pressão variável (média de $2,1 \mathrm{~kb}$, correspondendo a $7 \mathrm{~km}$ de profundidade); o fluido aquo-carbônico possui densidade global entre 0,73 e 0,97 $\mathrm{g} / \mathrm{cm}^{3}$, salinidade média de $6,6 \%$ em peso equivalente de $\mathrm{NaCl}$; e que a fração molar do $\mathrm{CO}_{2}\left(\mathrm{XCO}_{2}\right)$ varia entre 6 e 16 moles \%. Essas características são compatíveis com as de depósitos auríferos mesotermais / mesozonais estruturalmente controlados.

Palavras-chave: ouro, metalogênese, inclusões fluidas, $\mathrm{CO}_{2}$, Tapajós, Paleoproterozóico

INTRODUÇÃO Mais de uma centena de ocorrências e pequenos depósitos, ainda subeconômicos, distribuídos em pequenos distritos e campos mineralizados, foram descritos na província aurífera do Tapajós. Os depósitos estão hospedados em diferen- tes unidades paleoproterozóicas, compostas por rochas metavulcanossedimentares, gnaisses, rochas vulcânicas, gabros e, principal-mente, em diferentes gerações de granitóides de posicionamento tectônico e afinidades geoquímicas distintas (Klein 
et al. no prelo). A maioria dessas mineralizações é constituída por veios de quartzo alojados em estruturas rúpteis a rúptil-dúcteis e muitas se relacionam a um sistema transcorrente sinistral que delineou a principal confi-guração estrutural regional (Santos 1999, 2000, Klein et al. 2002).

Modelos metalogenéticos regionais foram desenvolvidos por Coutinho et al. (2000) e Santos et al. (2001). Coutinho et al. (2000) classificaram os depósitos como orogênicos (no sentido de Groves et al. 1998) com características mesozonais a epizonais e determinaram duas épocas de mineralização, baseados em idades-modelo em sulfetos, ocorridas em 1,96 Ga e 1,88 Ga. Santos et al. (2001) propuseram duas classes de depósitos: orogênicos e relacionados a intrusões. Os depósitos orogênicos foram subdivididos em associados a turbiditos e a arcos magmáticos, os primeiros hospedados em estruturas dúcteis em rochas metavulcanossedimentares do Grupo Jacareacanga, e os segundos hospedados em estruturas dúcteis-rúpteis em gnaisses (Complexo Cuiú-Cuiú) e granitóides (Suíte Tropas). Ambos apresentariam características mesozonais. Os depósitos relacionados a intrusões foram subdivididos em veios epizonais, posicionados em estruturas rúpteis que cortam as suítes granitóides Creporizão, Parauari e Maloquinha, e em disseminações epizonais e stockworks hospedados em rochas máficas e sedimentares, com características similares às dos depósitos tipo pórfiro. Santos et al. (2001) defendem apenas uma época metalogenética, que teria ocorrido em torno de 1,86 Ga. Klein et al. (2002) sustentam que, pelo menos para a porção sudeste da província (onde está inserido o prospecto Patinhas), um evento mineralizador teria ocorrido entre 1,97 e 1,95 Ga, durante deformação transcorrente (ver evolução estrutural abaixo) e estágios finais de posicionamento de granitóides pós-colisionais (Vasquez et al. 2002) da Suíte Intrusiva Creporizão. Nesta porção da província, os depósitos estão encaixados em gnaisses e em granitóides orogênicos e mostram evidências de posicionamento mesozonal a epizonal e de formação / deformação em condições rúpteis-dúcteis (eventualmente dúcteis). Esses depósitos diferem estrutural e texturalmente daqueles formados por volta de 1,88 a 1,86 Ga, bastante comuns no restante da província, e que apresentam características epizonais a epitermais (Dreher et al. 1998, Corrêa-Silva et al. 2001, Nunes et al. 2001, Klein et al. no prelo) e estão hospedados em granitóides pós-orogênicos (Suíte Maloquinha) e em rochas vulcânicas félsicas.

O prospecto Patinhas é um representante dos depósitos formados na região sudeste da província aurífera do Tapajós no que se refere à constituição dos depósitos, controle estrutural e elementos texturais e estruturais dos veios mineralizados (Klein et al. 2002). Este trabalho objetiva a caracterização das condições físico-químicas ( $\mathrm{P}, \mathrm{T}$, composição) do fluido responsável pela mineralização nesse prospecto, através do estudo petrográfico e microtermométrico de inclusões fluidas, o que auxiliará, juntamente com os dados paragenéticos, estruturais e texturais, no estabelecimento de um modelo deposicional para essa ocorrência aurífera. Trata-se de uma concentração mineral atualmente não econômica, mas os processos que atuaram na sua formação são os mesmos que aqueles que geram depósitos economicamente mais interessantes, de forma que informações sobre a gênese dessas pequenas concentrações são igualmente importantes (Skinner 1997). Sua importância maior reside na discussão e avaliação da influência de modificações e/ou re-equilíbrio das inclusões fluidas em situação de deformação dúctil acompanhando o seu aprisionamento.

GEOLOGIA REGIONAL A província aurífera do Tapajós loca- liza-se na porção central do cráton Amazônico (Fig. 1). Os gnaisses do Complexo Cuiú-Cuiú constituem a unidade aflorante mais antiga da região, com idades entre 2033 e $2005 \mathrm{Ma}$ (Santos et al. 2000, 2001). O Grupo Jacareacanga, de natureza metavulcanossedimentar, previamente posicionado no mesmo intervalo de idade, mostra cristais detríticos de zircão com idades variando entre $2190 \mathrm{Ma}$ e $1971 \mathrm{Ma}$ (Almeida et al. 2001b), o que sugere que essa unidade seja um pouco mais jovem, ou que existam outras seqüências metassedimentares na área. Essas unidades metamórficas foram intrudidas por diferentes gerações de granitóides, como as suítes intrusivas Creporizão, de 1,99-1,96 Ga (Ricci et al. 1999, Santos et al. 2000), Tropas, de 1,90 Ga (Santos et al. 2001), Parauari, de 1,891,88 Ga (Vasquez et al. 1999, Santos et al. 2000), além de rochas gabróicas de 1,87 Ga (Santos et al. 2001), e recobertas por sedimentação intra-arco a 1,90-1,89 Ga (Santos et al. 2001). Esse período orogênico foi seguido de intenso plutonismo e vulcanismo félsico entre 1,88 e 1,87 Ga (Vasquez et al. 1999, Santos et al. 2000, Lamarão et al. 2001).

A evolução estrutural da província é resumida a partir das propostas de Santos (2000), Almeida et al. (2001a), Santos et al. (2001) e Klein et al. (2002). O estágio inicial (D1), compressivo, gerou o bandamento gnáissico das rochas do Complexo Cuiú-Cuiú e a xistosidade das rochas do Grupo Jacareacanga, orientadas aproximadamente segundo $\mathrm{N}-\mathrm{S}$, e ocorreu provavelmente entre $2005 \mathrm{Ma}$ e $1997 \mathrm{Ma}$. O segundo estágio (D2) é fruto de regime transcorrente NW-SE, provavelmente entre $1997 \mathrm{Ma}$ e $1957 \mathrm{Ma}$, concomitantemente com a intrusão dos granitóides da Suíte Intrusiva Creporizão. Esse evento afetou os granitóides dessa unidade, transpôs as estruturas geradas em Dl e a ele se associam mineralizações auríferas como a do prospecto Patinhas. Um terceiro estágio (D3), também transcorrente, mas com estruturas NNESSW, afetou especialmente granitóides da Suíte Tropas, entre 1894 Ma e 1883 Ma. Evento posterior, eminentemente rúptil, gerou estruturas e / ou reativou estruturas mais antigas durante o posicionamento de granitóides relacionados a tectônica de distensão.

GEOLOGIA DO PROSPECTO PATINHAS A porçãosudeste da Província Aurífera do Tapajós, onde se localiza o prospecto Patinhas, é dominada pelos gnaisses do Complexo Cuiú-Cuiú e granitóides da Suíte Intrusiva Creporizão, ambos intrudidos por granitóides da suíte Parauari e cobertos, ao sul, por rochas sedimentares da Formação Buiuçu (Fig. 1). Nessa região são conhecidas mais de vinte ocorrências auríferas primárias em veios de quartzo, com características similares (Klein et al. 2002).

A mineralização aurífera do Patinhas está associada a um veio de quartzo posicionado longitudinalmente em uma zona de cisalhamento que corta um hornblenda-gnaisse granodiorítico de granulação média, pertencente ao Complexo Cuiú-Cuiú, foliado segundo N-S a NNW-SSE. A zona de cisalhamento hospedeira é transcorrente, subvertical, orientada segundo $\mathrm{N} 20^{\circ}-30^{\circ} \mathrm{E}$ e tem caráter rúptil-dúctil (Rosa-Costa \& Carvalho 1999). O veio possui espessura média de $70 \mathrm{~cm}$ e seus contatos com a rocha encaixante são marcados por estreito halo hidrotermal e pela presença de foliação milonítica desenvolvida sobre essa rocha (Fig. 2). O quartzo é do tipo leitoso e lateralmente apresenta variações estruturais. Próximo ao contato com a rocha encaixante mostra planos pouco espaçados (Fig. 3A) que refletem a deformação dúctil melhor desenvolvida na rocha encaixante. Em porções mais interiores, a estrutura torna-se maciça (Fig. 3B). Esses aspectos sugerem que o veio posicionou-se em profundidades, pelo menos, moderadas 
(Vearncombe 1993), em estrutura ativa, cuja atividade estendeu-se até algum tempo após a formação do veio. $\mathrm{O}$ caráter maciço e a ausência de textura laminada são, por sua vez, indicativos de que mecanismo cíclico de válvula não operou, tendo o veio se formado provavelmente em um só estágio de dilatação e selagem da estrutura hospedeira (p. ex. Cox 1995).

A alteração hidrotermal, pós-metamórfica, está superposta por alteração supergênica, de forma que não pode ser avaliada satis-

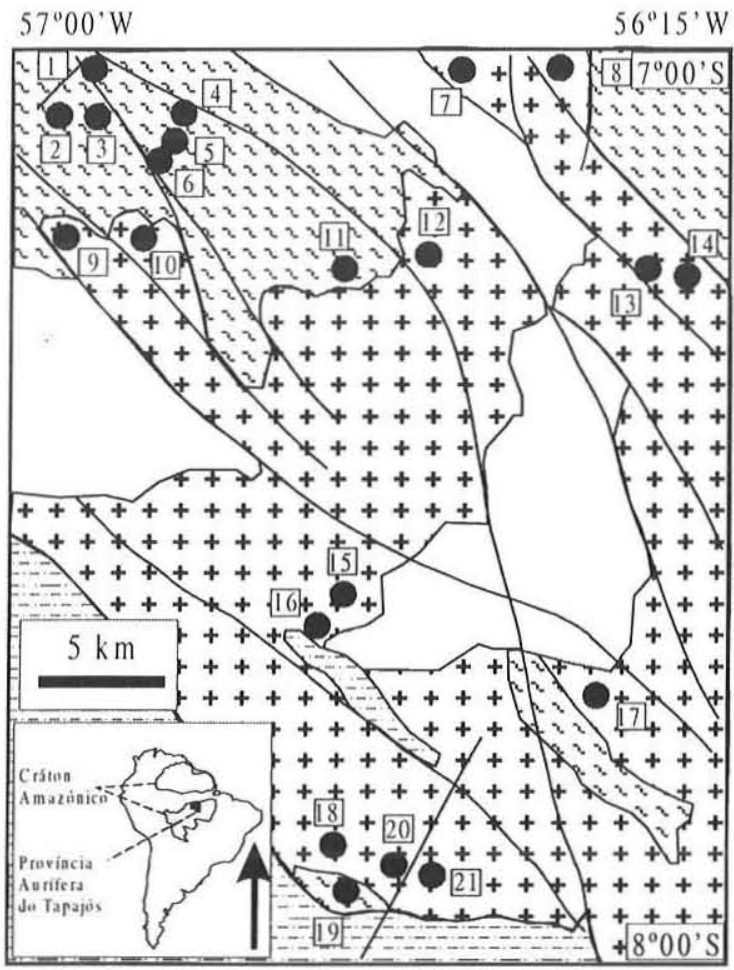

Paleoproterozóico

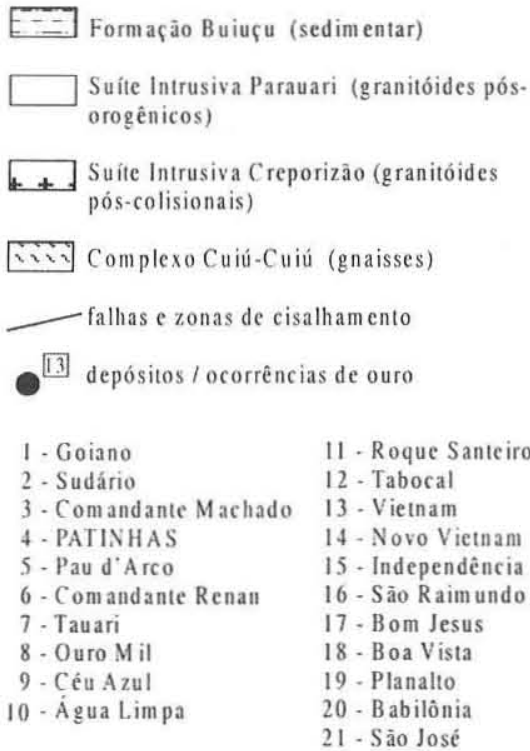

Figura I - Mapa geológico de parte da província aurífera Tapajós,com a localização do prospecto Patinhas e demais ocorrências auriferas na área. Modificado de Klein et al. (2002). fatoriamente. Entretanto, quartzo e feldspato de granulação grossa, e algum epidoto e pirita encontram-se ainda preservados e são observados em vênulas muito finas. Os sulfetos estão preferencialmente disseminados junto ao contato veio-encaixante ou ocorrem, já oxidados, em planos sinuosos que margeiam o veio de quartzo principal (Fig. 2B). Localmente, formam agregados e percentuais volumétricos importantes na porção mais central do veio (Fig. 3B). Pirita é o sulfeto dominante, ocorrendo também calcopirita em porções muito subordinadas e a covelita como produto de alteração secundária da calcopirita. Partículas anédricas de ouro, preenchendo microfraturas em cristais de pirita (Fig. 4), são observadas ao microscópio.

\section{ESTUDODEINCLUSÕESFLUIDAS O estudo petrográ-} fico e microtermométrico de inclusões fluidas foi realizado na porção maciça e rica em sulfetos do veio de quartzo (Fig. 3B), interpretada

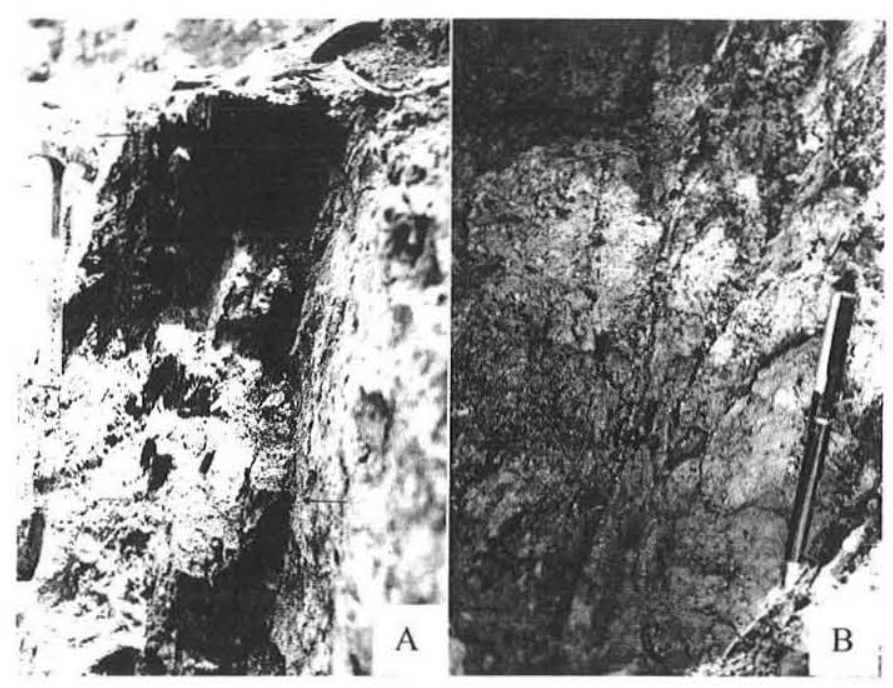

Figura 2 - A) Vista de parte do veio de quartzo aurífero; B) detalhe dos planos sinuosos que margeiam o veio mineralizado (segundo Rosa-Costa \& Carvalho 1998).

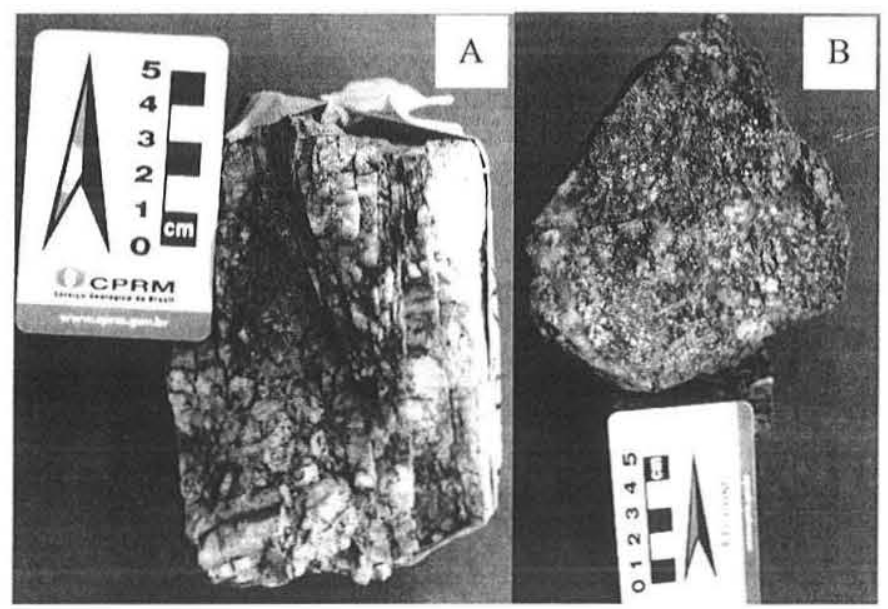

Figura 3 - Aspectos texturais e estruturais do veio de quartzo aurífero. A) veio pobre em sulfetos, foliado no contato com a rocha encaixante; $B$ ) veio de quartzo maciço (cinza claro) rico em sulfetos (cinza escuro). 
como tendo sido mais preservada da deformação dúctil que atingiu as bordas do veio. As análises microtermométricas foram realizadas no Laboratório de Metalogênese da Universidade Federal do Pará, utilizando-se platina Chaixmeca. Preparação de amostras e procedimentos analíticos e interpretativos seguiram recomendações de Roedder (1984). Calibração foi realizada com padrões de $\mathrm{CO}_{2}$ e $\mathrm{H}_{2} \mathrm{O}$ disponíveis. A precisão das medidas é estimada em \pm $0,5^{\circ} \mathrm{C}$ para temperaturas inferiores $\mathrm{a}-20^{\circ} \mathrm{C}, \pm 0,3^{\circ} \mathrm{C}$ para o intervalo entre $-20^{\circ}$ e $40^{\circ} \mathrm{C} \mathrm{e} \pm 3^{\circ} \mathrm{C}$ para temperaturas superiores a $40^{\circ} \mathrm{C}$. Salinidades foram calculadas a partir das temperaturas de fusão do clatrato (Collins, 1979) e do gelo (Bodnar, 1992). Densidades foram calculadas pelas equações de Bowers \& Helgeson (1983) e Brown \& Lamb (1986), respectivamente para inclusões portadoras de $\mathrm{CO}_{2}$ e inclusões aquosas.

Petrografia Ao microscópio, o quartzo tem dimensões variadas, com cristais maiores envoltos por matriz recristalizada muito fina (Fig. 5). Os cristais maiores contêm efeitos variáveis de deformação dúctil, como extinção ondulante moderada a forte, lamelas de deformação, bordas com contatos serrilhados, desenvolvimento de subgrãos e porções recristalizadas, pobres em, ou desprovidas de, inclusões fluidas. Esse comportamento é devido à deformação que acompanhou e sucedeu a formação do veio de quartzo.

Enxames de inclusões fluidas ocorrem no quartzo, tanto em triIhas bem definidas como manteando cristais recristalizados (Fig. 6A). Outras inclusões fluidas ocorre em áreas menos deformadas e/ou reliquiares dos cristais de quartzo, de forma isolada ou em pequenos grupamentos (Fig. 6A e B). Estas inclusões, provavelmente mais precoces e/ou mais preservadas, foram utilizadas para a obtenção dos dados microtermométricos, tendo sido identificados três tipos principais, com base nas proporções entre as fases fluidas em temperatura ambiente (Fig. 7) e no seu comportamento sob resfriamento e aquecimento. Todas apresentam dimensões bastante reduzidas, tipicamente entre $5 \mu \mathrm{me} 10 \mu \mathrm{m}$.

As inclusões do tipo I (carbônicas) são ricas em $\mathrm{CO}_{2}$ e monoou bifásicas na temperatura ambiente. Têm formas variadas, regulares ou não, e se distribuem de forma randômica ou em peque-nos grupamentos, geralmente associadas com as do tipo 2 .

Inclusões do tipo 2 contêm líquido aquoso salino e $\mathrm{CO}_{2}$ (aquocarbônicas). São bi- ou trifásicas em temperatura ambiente, com a fase $\mathrm{CO}_{2}$ ocupando entre 20 e $70 \%$ do volume total das cavidades, excepcionalmente $\sim 90 \%$, quando apresentam um quase imperceptível filme de $\mathrm{H}_{2} \mathrm{O}$ entre a bolha de $\mathrm{CO}_{2}$ e a parede das inclusões. Exibem formas dominantemente irregulares a elipsoidais e distribuem-se da mesma maneira que as inclusões tipo 1 .

O tipo 3 consiste em inclusões aquosas bifásicas e divide-se em dois subtipos, em função de sua relação com os outros tipos e forma de ocorrência. $\mathrm{O}$ subtipo $3 \mathrm{~A}$ associa-se espacialmente a inclusões dos tipos 1 e 2; o subtipo 3B ocorre em trilhas estreitas posteriores aos demais tipos. $\mathrm{O}$ grau de preenchimento das inclusões pertencentes ao subtipo $3 \mathrm{~B}$ tende a ser, em geral, maior $(0,90$ a 0,95$)$ do que o das inclusões $3 \mathrm{~A}(0,8$ a 0,95$)$.

Resultados microtermométricos TIPOS l e 2 Esses tipos, além de ocorrerem em constante associação, apresentam resultados microtermométricos semelhantes (Tabela 1), de modo que são descritos em conjunto. A temperatura de fusão da fase carbônica $\left(\mathrm{Tf}_{\mathrm{CO} 2}\right.$ ) variou de $-58,2^{\circ} \mathrm{C}$ a $-56,6^{\circ} \mathrm{C}$, mas com forte concentração de valores próximos ao ponto de fusão do $\mathrm{CO}_{2}$ puro $\left(-56,6^{\circ} \mathrm{C}\right)$ (Fig. $8 \mathrm{~A})$. Isto indica que $\mathrm{CO}_{2}$ é o principal componente da fase carbônica, mas percentuais subordinados a moderados de outros com-

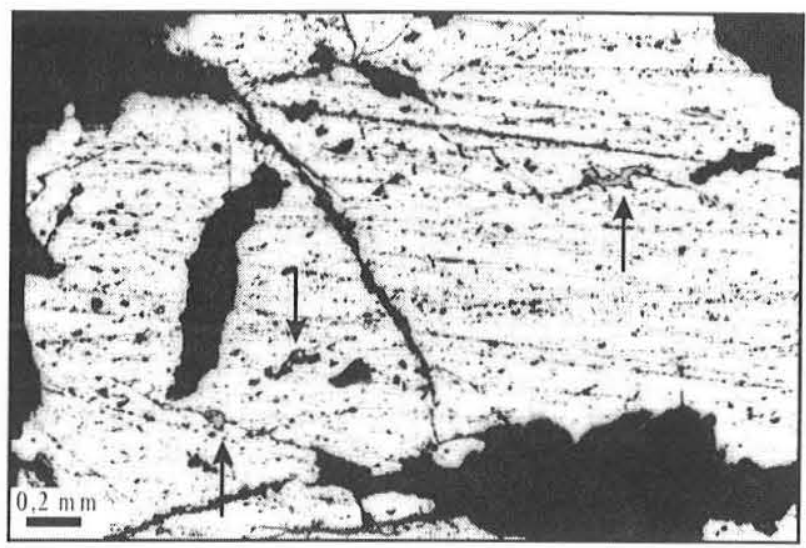

Figura 4 - Fotomicrografia de cristal de pirita (cinza claro) contendo partículas de ouro (setas) em microfraturas.

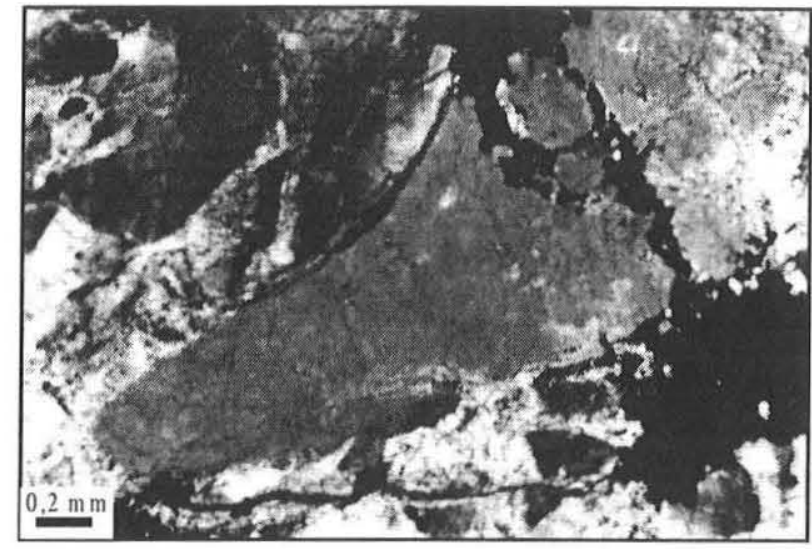

Figura 5 - Fotomicrografia (nicóis cruzados) mostrando aspectos texturais do quartzo de veio. Cristal reliquiar mais límpido (centro) rodeado por cristais com forte extinção ondulante (vértice superior esquerdo) e por mosaicos de subgrãos recristalizados (base da foto).

ponentes (p. ex., $\mathrm{CH}_{4}$ e / ou $\mathrm{N}_{2}$ ) estão presentes num grupo menor de inclusões. A temperatura de homogeneização parcial da fase carbônica $\left(\mathrm{Th}_{\mathrm{CO} 2}\right)$ tem variação moderada, entre $21,8^{\circ} \mathrm{C}$ e $31,1^{\circ} \mathrm{C}$, com moda acentuada em $30,5^{\circ} \mathrm{C}$ (Fig. 8B). A densidade do $\mathrm{CO}_{2}$, calculada a partir dos valores de $\mathrm{Th}_{\mathrm{CO} 2}$, mostra valores moderados, entre 0,47 e $0,75 \mathrm{~g} / \mathrm{cm}^{3}$, enquanto que a densidade global das inclusões do tipo 2, também moderada, situa-se entre 0,73 e 0,97 g/ $\mathrm{cm}^{3}$. As frações molares $\left(\mathrm{XCO}_{2}\right)$ das inclusões dos tipos 1 e 2 são estimadas entre 66 e 71 moles \% e entre 6 a 16 moles \%, respectivamente. Clatratos, formados durante o resfriamento das inclusões tipo 2, fundem-se num intervalo relativamente estreito de temperatura, entre $4,1^{\circ} \mathrm{C} \mathrm{e} 7,2^{\circ} \mathrm{C}$ (Fig. $8 \mathrm{C}$ ), indicando salinidade média de $6,6 \%$ em peso equivalente de $\mathrm{NaCl}$. A homogeneização final desse mesmo tipo de inclusão ocorre sempre na fase líquida, em temperaturas (Tht) entre $307^{\circ} \mathrm{Ce} 389^{\circ} \mathrm{C}$ (Fig. 8D).

TIPO 3 O ponto eutético para os dois subtipos apresenta variado e semelhante intervalo de temperaturas, entre $-43^{\circ} \mathrm{C} \mathrm{e}$ $24^{\circ} \mathrm{C}$, indicando o aprisionamento de outros cátions, além do $\mathrm{Na}$. Nenhuma relação é observada entre as temperaturas do eutético e 


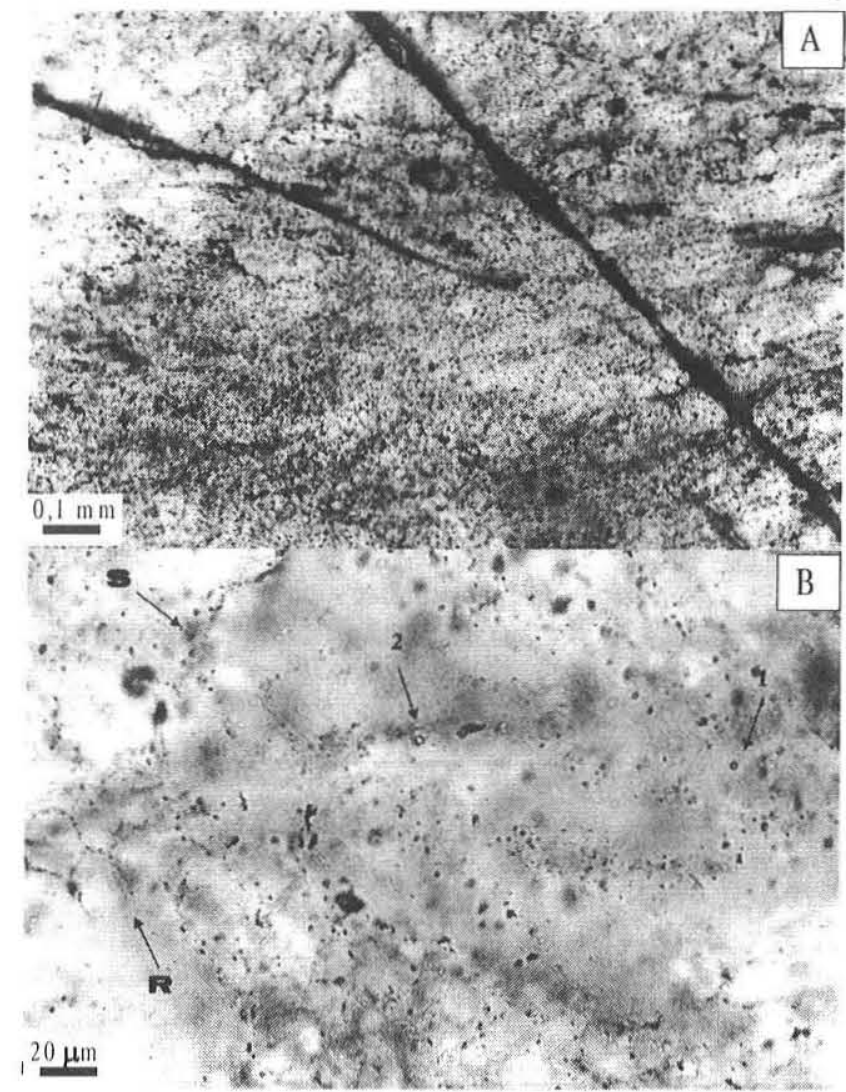

Figura 6 - Fotomicrografias mostrando relações texturais das inclusões fluidas no quartzo de veio aurífero. A) grande cristal de quartzo com enxames de inclusões fluidas secundárias (centro), contrastando com cristal mais límpido (vértice superior esquerdo) com grupamentos de inclusões aleatórias (seta); B) grande cristal de quartzo (centro) com inclusões isoladas dos tipos 1 e 2 (setas), manteado por inclusões secundárias $(S)$ e margeado por pequenos cristais euédricos (hexagonais) recristalizados $(R)$, também manteados por inclusões secundárias.

de fusão final do gelo. As inclusões do subtipo $3 \mathrm{~A}$ apresentam a fusão do gelo entre $-12^{\circ} \mathrm{Ce}-0,1^{\circ} \mathrm{C}$ (Fig. $8 \mathrm{C}$ ), o que indica salinidades inferiores a $6,5 \%$ em peso equivalente de $\mathrm{NaCl}$ e densidades entre $0,70 \mathrm{~g} / \mathrm{cm}^{3} \mathrm{e} 1,05 \mathrm{~g} / \mathrm{cm}^{3}$. A homogeneização final ocorre tipicamente entre $273^{\circ} \mathrm{Ce} 319^{\circ} \mathrm{C}$, intervalo que se superpõe ao limite inferior de Tht das inclusões do tipo 2 (Fig. 8D), com as quais comumente apresentam associação espacial. O caráter texturalmente tardio das inclusões do subtipo 3B é confirmado pelos dados microtermométricos, que mostram Tht inferiores às do subtipo $3 \mathrm{~A}$, entre $112^{\circ} \mathrm{Ce} 243^{\circ} \mathrm{C}$ (Fig. 8D). A salinidade dessas inclusões é mais variável, atingindo valores de até $21 \%$ em peso equivalente de $\mathrm{NaCl}$, fornecidos por fusões do gelo em até $-19^{\circ} \mathrm{C}$ (Fig. 8C).

DISCUSSÃO E CONCLUSÕES O veio de quartzo aurífero do prospecto Patinhas está hospedado em zona de cisalhamento transcorrente rúptil-dúctil. As relações entre o veio, a estrutura hospedeira e a rocha encaixante (gnaisse), as estruturas presentes no veio e na rocha encaixante e aspectos petrográficos do quartzo indicam que o posicionamento de veio ocorreu em profundidades

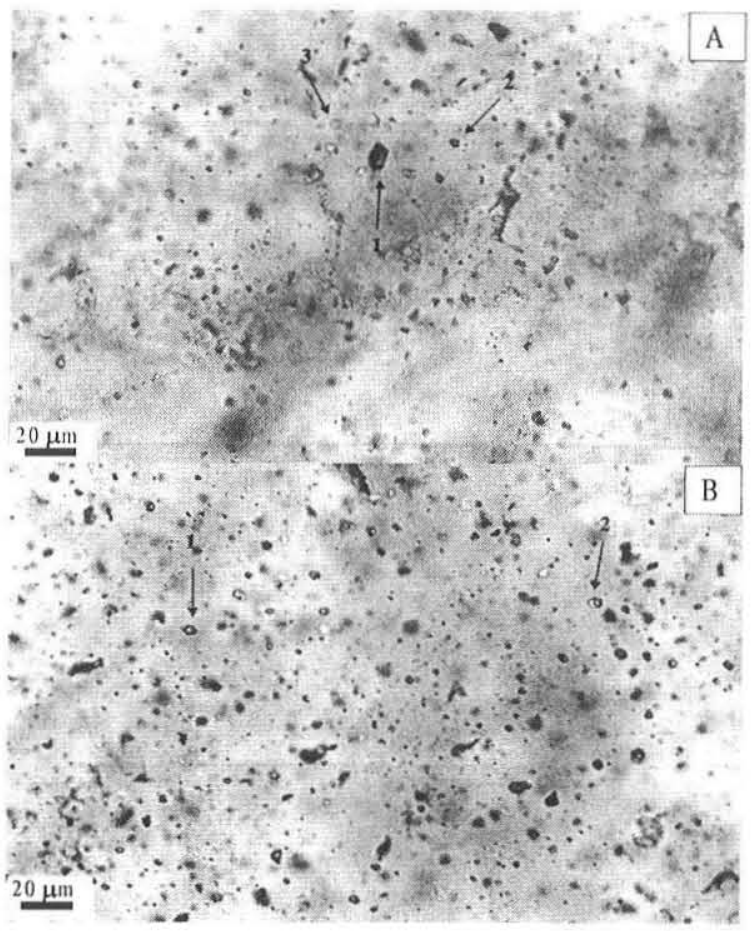

Figura 7 - Fotomicrografias mostrando a distribuição dos tipos de inclusões fluidas em veio de quartzo aurífero. A) associação espacial entre inclusões dos tipos 1, 2 e 3A (setas); uma trilha de inclusões secundárias tardias (tipo $3 B$ ) corta o quartzo do vértice inferior esquerdo para o superior direito; no centro, à direita, uma inclusão grande com claras evidências de estrangulamento. B) inclusões dos tipos 1 e 2, distribuidas aleatoriamente e com proporções variáveis entre $\mathrm{CO}_{2}$ e $\mathrm{H}_{2} \mathrm{O}$.

Tabela I - Resumo dos resultados microtermométricos.

\begin{tabular}{|c|c|c|c|c|}
\hline & tipo 1 & tipo 2 & tipo $3 \mathrm{~A}$ & tipo 3B \\
\hline $\mathrm{Tf}_{\mathrm{CO} 2}\left({ }^{\circ} \mathrm{C}\right)$ & $-58,2$ a $-56,6$ & $-58,0$ a $-56,6$ & - & - \\
\hline $\mathrm{Th}_{\mathrm{CO} 2}\left({ }^{\circ} \mathrm{C}\right)$ & 21,8 a 31,1 & 28,0 a 31,1 & - & - \\
\hline Tclat $\left({ }^{\circ} \mathrm{C}\right)$ & - & 4,1 a 7,2 & - & - \\
\hline Tht $\left({ }^{\circ} \mathrm{C}\right)$ & - & 307 a 389 & 255 a 319 & 112 a 243 \\
\hline Teu $\left({ }^{\circ} \mathrm{C}\right)$ & - & - & $-43 a-24$ & $-43 a-24$ \\
\hline $\operatorname{Tfg}\left({ }^{\circ} \mathrm{C}\right)$ & - & - & $-12 \mathrm{a}-0,1$ & $-19 a-0,1$ \\
\hline
\end{tabular}

$\mathrm{Tf}_{\mathrm{CO} 2}=$ temperatura de fusão da fase carbônica; $\mathrm{Th}_{\mathrm{CO} 2}=$ temperatura de homogeneização da fase carbônica; Tclat - temperatura de fusão do clatrato; Tht - temperatura de homogeneização total: Teu = temperaturra do eutético; Tfg: = temperatura de fusão do gelo

moderadas na crosta, algo acima da transição rúptil-dúctil (6-10 $\mathrm{km}$ ). Esses dados também sugerem que a estrutura encontrava-se ativa durante o posicionamento do veio e que a movimentação se prolongou até depois da precipitação do mesmo. Microtexturas mostram que o quartzo foi afetado por deformação dúctil de intensidade variada, incluindo recristalização dinâmica.

Três tipos fundamentais de inclusões fluidas - carbônicas, aquocarbônicas e aquosas - foram aprisionadas principalmente em domínios menos afetados pela deformação, mas são registrados também, com as mesmas características, em domínios recristalizados. Relações texturais e dados microtermométricos indicam que as 

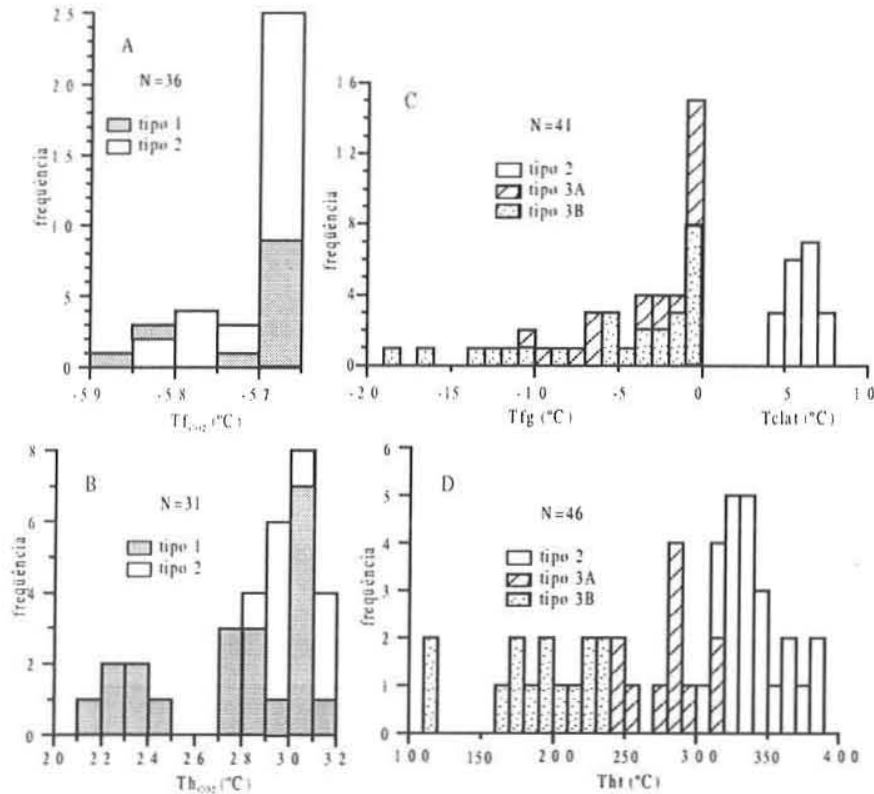

Figura 8 - Histogramas mostrando a distribuição de freqüência das propriedades microtermométricas das inclusões fluidas. A) temperatura de fusão da fase carbônica $\left(T f_{\left(\mathrm{C}_{2}\right.}\right)$; B) temperatura de homogeneização da fase carbônica (Th C) temperaturas de fusão de clatratos (Tclat) em inclusões do tipo 2 e do gelo (Tfg) em inclusões do tipo 3; D) temperaturas de homogeneização total (Tht).

inclusões fluidas do tipo 3B (aquosas) são tardias, envolvendo mais de uma geração de fluidos aprisionados em condições rúpteis, retrogressivas, talvez durante o soerguimento regional, não tendo relação com a mineralização aurífera. Os demais tipos, mais precoces, distribuem-se de forma isolada ou coexistente, em porções do mineral hospedeiro menos afetadas por deformação dúctil. Estes tipos encontram-se no estado heterogêneo (imiscibilidade) e mostram um completo espectro composicional entre os termos extre$\operatorname{mos} \mathrm{CO}_{2}$ e $\mathrm{H}_{2} \mathrm{O}$ (+sais): $\mathrm{CO}_{2}$ (tipo 1); $\mathrm{CO}_{2} \gg \mathrm{H}_{2} \mathrm{O}$ (tipo 2); $\mathrm{H}_{2} \mathrm{O}>$ $\mathrm{CO}_{2}$ (tipo 2); e H,O (tipo $3 \AA$ ). Dados microtermométricos como $\mathrm{Th}_{\mathrm{CO} 2}$ (densidade) e Tht mostram certa variação, enquanto que a salinidade é menos variável. Essas feições podem ser produzidas pela ação individual ou combinada de processos tão distintos quanto separação de fases, mistura de fluidos e modificações ocorridas durante e/ou após o aprisionamento.

Para o tipo de depósito aurífero em estudo, as variações descritas para as inclusões fluidas são mais comumente atribuídas à separação de fases a partir de fluido aquo-carbônico, freqüentemente desencadeada por flutuações de pressão (Robert \& Kelly 1987). Embora os aspectos texturais e estruturais do veio e da rocha encaixante imediata sugiram ausência de mecanismo cíclico de válvula, a coexistência de feições dúcteis e rúpteis indicam que variações de pressão ocorreram.

Dentre os critérios estabelecidos por Ramboz et al. (1982) para justificar imiscibilidade, a coexistência e contemporaneidade de fluidos com composições contrastantes é texturalmente demonstrada e aprisionamento heterogêneo é documentado. Não se observa a homogeneização das inclusões com menor e maior proporção de vapor para as fases líquido e vapor, respectivamente.

Mistura de fluido carbônico anidro ou aquo-carbônico, mais quentes, com fluido aquoso mais frio pode gerar todo o espectro composicional observado (Anderson et al. 1992). Isto requer correlação entre temperatura e salinidade (Cathelineau \& Marignac 1994), mas a variação de salinidade observada é pequena (Fig. 9), o que pode ser devido à separação de fases, enquanto que mistura provocaria variações mais significativas (Wilkinson 2001).

Devido ao caráter sintectônico, com relação à estrutura hospedeira, e às evidências de deformação identificadas no veio de quartzo aurífero, o reequilíbrio das inclusões sob diferentes condições de pressão e temperatura e modificações ocorridas após a sua formação precisam ser levadas em consideração. Um número expressivo de inclusões ocorre em limites de grãos, tanto em cristais maiores, como entre subgrãos (Figs. 6e 7) desenvolvidos durante a recuperação e / ou recristalização dinâmica, o que pode ser devido à captura de fluidos durante migração de bordas de cristais (Klemd 1998). Feições de estrangulamento (Fig. 7A) são comuns e texturas de implosão e explosão também ocorrem. Entretanto, essas inclusões não foram utilizadas nas análises microtermométricas, mas sim, aquelas inclusões de formas mais regulares e, possivelmente, mais preservadas de alterações subseqüentes ao aprisionamento.

Em situações fluido-dinâmicas similares às encontradas no Patinhas, é comum o relato de intensa extração seletiva de $\mathrm{H}_{2} \mathrm{O}$, deixando um predomínio $(>80 \%)$ de inclusões carbônicas (Schwartz et al. 1992). A remoção preferencial de $\mathrm{H}_{2} \mathrm{O}$ seria induzida por deformação cristal-plástica ou recristalização dinâmica do quartzo (Hollister 1990, Hall \& Sterner 1993), difusão (Bakker \& Jansen 1991), maior mobilidade da $\mathrm{H}_{2} \mathrm{O}$ em relação ao $\mathrm{CO}_{2}$ devido ao contraste de densidades ou diferentes polaridades dos dois compostos (Crawford \& Hollister 1986). Contrariamente, no caso em estudo, inclusões aquosas são abundantes. No caso de remoção seletiva da $\mathrm{H}_{2} \mathrm{O}$, seria esperado que esse fluido fosse em grande parte eliminado durante a migração das bordas de grãos.

Em suma, as inclusões aqui estudadas não são consideradas primárias, no sentido de Roedder (1984), que teriam sido poupadas de eliminação durante deformação e recristalização. Alternativamente, são interpretadas como aprisionadas e re-equilibradas ao longo da evolução do sistema hidrotermal e da estrutura hospedeira, que, conforme a evidência textural e estrutural apresentada, ocorreu, pelo menos em parte, contemporaneamente à

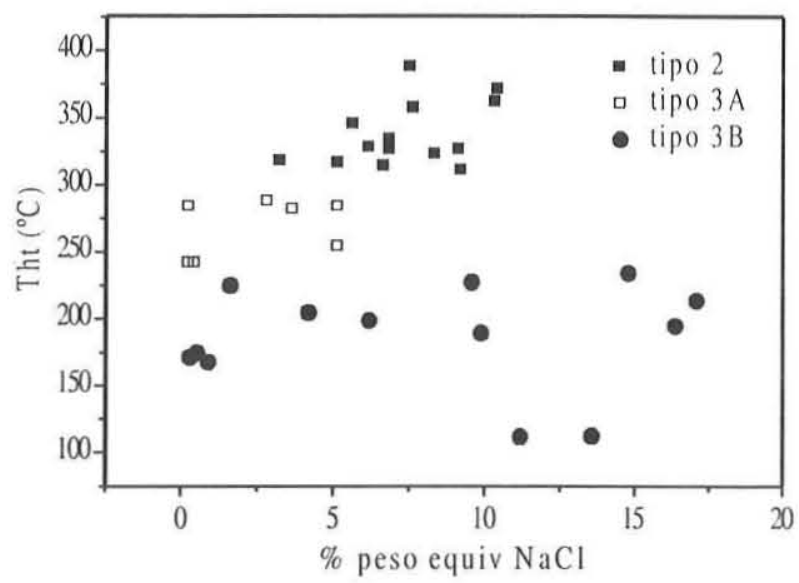

Figura 9 - Diagrama salinidade versus temperatura de homogeneização total (Tht) de inclusões fluidas aquosas $e$ aquo-carbônicas. Explicações no texto. 
mineralização (corrobora esta interpretação o fato de que, mesmo em cristais menores, recristalizados, algumas inclusões com dimensões suficientes para permitir a análise microtermométrica apresentam propriedades idênticas àquelas observadas em inclusões contidas nos núcleos dos grandes cristais). Em sua totalidade as inclusões foram aprisionadas em condições sin-deformacionais, provavelmente em um único episódio de infiltração de fluidos e representam, portanto, as condições de evolução termobarométrica e composicional do sistema hidrotermal-estrutural e da mineralização. Considera-se, ainda, que as inclusões fluidas tenham sido modificadas e re-equilibradas durante separação de fases que acompanhou a história dinâmica do veio (p. ex, Kolb et al. 2000). As inclusões aquo-carbônicas são as representantes mais prováveis do fluido original, sendo as inclusões carbônicas e aquosas produto do aprisionamento acidental de termos puros durante a separação de fases, localmente associadas com modificações posteriores.

Condições de temperatura e pressão da mineralização Assumindo a ocorrência de separação de fases como o mais indicado para a formação das inclusões descritas, essa ocorreu sobre ou próximo ao solvus do sistema químico em questão, de forma que as temperaturas de homogeneização devem representar as condições reinantes durante a mineralização, não necessitando de correção de pressão. Na ausência de geotermômetro independente, o procedimento usado para limitar as condições de P-T da mineralização é a inteerseção das isócoras representantes da maior e menor densidade das inclusões aquo-carbônicas com o solvus compatível com a composição do fluido (Hagemann \& Brown 1996). O solvus utilizado é o do sistema com $\mathrm{XCO}_{2}=10 \mathrm{~mol} \%$ e $6 \%$ em peso equivalente de $\mathrm{NaCl}$ (Bowers \& Helgeson 1983). Além disso, é utilizado o intervalo de variação das temperaturas de homogeneização registradas. Segundo esse procedimento, os limites de P-T da mineralização do Patinhas são $307^{\circ}$ a $389^{\circ} \mathrm{Ce} 1,0$ a $3,3 \mathrm{~kb}$ (Fig. 10). Como as inclusões foram aprisionadas em ambiente estrutural ativo, é provável que os dados médios de pressão representem condições de pressão média durante a mineralização (Hagemann \& Brown 1996). No caso estudado, isso significa cer- ca de $2,1 \mathrm{~kb}$, correspondendo a uma profundidade em torno de 7 km, o que está de acordo com as condições estruturais observadas.

As características tipológicas e do fluido no prospecto Patinhas são similares às de outros depósitos em veios de quartzo controlados estruturalmente e associados a ambientes orogênicos (p.ex., Groves et al., 1998) e enquadram-se no modelo de depósitos mesozonais orogênicos associados a arcos magmáticos, conforme proposta de Santos et al. (2001) para a província Tapajós.

Agradecimentos Aos revisores da RBG pelas sugestões ao manuscrito. Contribuição ao projeto PRONEX-CNPq-FADESP 66.2103/1998-0, processo n ${ }^{\circ} 420.00 / 00$.

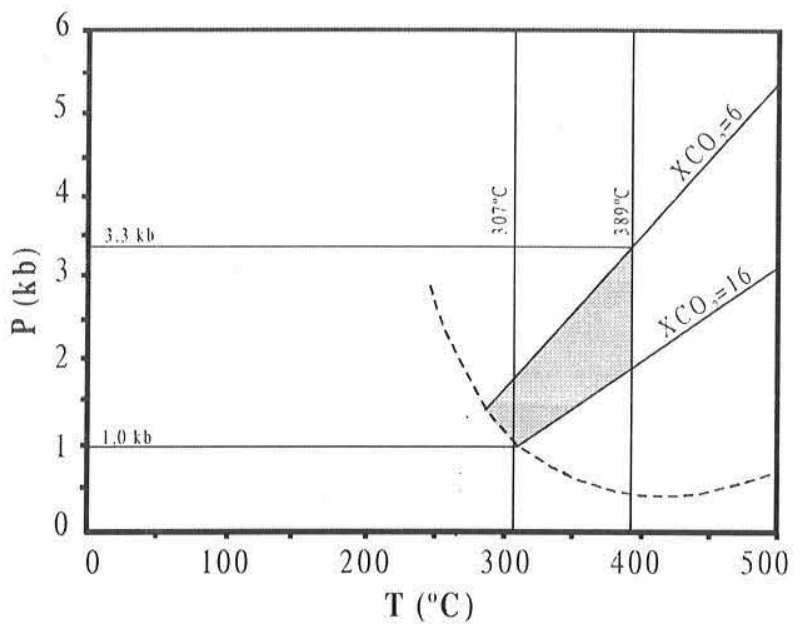

Figura 10 - Diagrama P-T mostrando isócoras para as frações molares (e densidades) extremas das inclusões aquocarbônicas $\left(\mathrm{XCO}_{2}=6\right.$ e 16) e o solvus para o sistema com $\mathrm{XCO}_{2}=10$ e 6,6\% peso de $\mathrm{NaCl}$ equiv. (Bowers \& Helgeson 1983). A área sombreada representa os limites de P-T estimados para a mineralização do Patinhas.

\section{Referências}

Almeida M.E., Ferreira A.L., Brito M.F.L., Monteiro M.A.S. 2001 a. Evolução tectono-estrutural da Província Tapajós com base na geologia das folhas Vila Mamãe Anã e Jacareacanga (1:250.000), região limite dos estados do Amazonas e Pará. In: Reis N.J., Monteiro M.A.S. (org). Manaus, SBG-NO, Contribuições à Geologia da Amazônia v. 2, 57-112.

Almeida M.E., Ferreira A.L., Macambira M.J.B., Sachett C.R.. 2001 b. Time constraint based on zircon dating for the Jacareacanga Group (Tapajós Province, Amazon Craton, Brazil). In: Simp. Sudamericano Geol. Isotopica, 3. Pucon, Chile. Actas (CD-ROM).

Anderson M.R., Rankin A.H., Spiro B. 1992. Fluid mixing in the generation of mesothermal gold mineralization in the Transvaal Sequence, Transvaal, South Africa. European J. Mineral., 4: 933-948.

Bakker R.J. \& Jansen B.H. 1991. Experimental post-entrapment water loss from synthetic $\mathrm{CO}_{2}-\mathrm{H}_{2} \mathrm{O}$ inclusions in natural quartz. Geochim. Cosmochim. Acta, 55: 2215-2230.

Bodnar R.J. 1992. Revised equation and table for freezing point depressions of $\mathrm{H}_{2} \mathrm{O}$-salt fluid inclusions. PACROFI, Fourth Biennial PanAmerican Conference on Research on Fluid Inclusions. Progr. and
Abstracts, v.4, p. 15.

Bowers T.S. \& Helgeson H.C. 1983. Calculation of the thermodynamic and geochemical consequences of nonideal mixing in the system $\mathrm{H}_{2} \mathrm{O}-\mathrm{CO}_{2}-\mathrm{NaCl}$ on phase relations in geologic systems: equation of state for $\mathrm{H}_{2} \mathrm{O}-\mathrm{CO}_{2}-\mathrm{NaCl}$ fluids at high pressures and temperatures. Geochim. Cosmochim. Acta, 47: 1247-1275.

Brown P.E. \& Lamb W.M. 1986. Mixing of $\mathrm{H}_{2} \mathrm{O}-\mathrm{CO}_{2}$ in fluid inclusions. Geobarometry and Archean gold deposits. Geochim. Cosmochim. Acta, 50: 847-852.

Cathelineau M. \& Marignac C. 1994. Use of fluid inclusions for a better understanding of intracontinental geothermal activities. In: De Vivo B. \& Frezzotti M.L. (eds.) Fluid inclusions in minerals: methods and applications. Virginia Tech, Blacksburg, pp. 309-326.

Collins P.L.F. 1979. Gas hydrates in $\mathrm{CO}_{2}$-bearing fluid inclusions and the use of freezing data for estimation of salinity. Econ. Geol., 74, 14351444.

Corrêa-Silva R.H., Juliani C., Bettencourt J.S., Nunes C.M.D., Almeida T.I.R. 2001. Caracterização de um sistema epitermal low-sulfidation 
(ou adulária-sericita) hospedado em vulcânicas e vulcanoclásticas do Grupo Iriri na Província aurífera do Tapajós (PA). In: SBG-NO, Simp. Geol. Amazônia, 7, , Resumos expandidos (CD-ROM).

Coutinho M.G.N., Santos J.O.S., Fallick A.E., Lafon J.M. 2000. Orogenic gold deposits in Tapajós Mineral Province, Amazon, Brazil. In: International Geological Congress, 31, Rio de Janeiro, abstracts (CD-ROM)

Cox S.F. 1995. Faulting processes at high fluid pressures: an example of fault valve bahavior from the Wattle Gully Fault, Victoria, Australia J. Geophys. Res., 100 (B7): 12841-12859.

Crawford M.L. \& Hollister L.S. 1986. Metamorphic fluids: the evidence from fluid inclusions. In: Walther J.V. \& Wood B.J. (eds.) Fluid rock interaction during metamorphism. Physical Geochemistry, Springer, Berlin, v 5, 1-35.

Dreher A.M., Vlach S., Martini S.L. 1998. Adularia associated with epithermal gold veins in the Tapajós Mineral Province, Pará State, Northern Brazil. Rev. Bras. Geoc., 28: 397-404.

Groves D.I., Goldfarb R.J., Gebre-Mariam M., Hagemann S., Robert F. 1998. Orogenic gold deposits: a proposed classification in the context of their crustal distribution and relationship to other gold deposits types. Ore Geol. Reviews, 13: 7-27.

Hagemann S.G. \& Brown P.E. 1996. Geobarometry in Archean lode-gold deposits. European J. Mineral., 8: 937-960.

Hall D.L. \& Sterner S.M. 1993. Preferential water loss from synthetic fluid inclusions. Contrib. Mineral.Petrol., 114: 489-500.

Hodgson C.J. 1989. The structure of shear-related, vein-type gold deposits: a review. Ore Geol. Reviews, 4: 231-273.

Hollister L.S. 1990. Enrichment of $\mathrm{CO}_{2}$ in fluid inclusions in quartz by removal of $\mathrm{H}_{2} \mathrm{O}$ during crystal-plastic deformation. J. Struct. Geol., 12: 895-901.

Klein E.L., Santos R.A., Fuzikawa K., Angélica R.S. 2001. Hydrothermal fluid evolution and structural control of the brittle-style Guarim lode-gold mineralisation, Tapajós Province, Amazonian Craton, Brazil. Mineral. Depos., 36: 149-164.

Klein E.L., Vasquez M.L., Rosa-Costa L.T., Carvalho J.M.A. 2002. Geology of Paleoproterozoic gneiss- and granitoid-hosted gold mineralization in Southern Tapajós Province, Amazonian Craton, Brazil. Int. Geol.Review, 44: 544-558.

Klein E.L., Almeida M.E., Vasquez M.L., Bahia R.B.C., Quadros M.L.E.S., Ferreira A.L. (no prelo) Geologia e recursos minerais da Província Mineral do Tapajós, Estados do Pará e Amazonas, Escala I: 500.000. CPRM

Klemd R. 1998. Comment on the paper by Schmidt Mumm et al. High $\mathrm{CO}$, content of fluid inclusions in gold mineralisations in the Ashanti Belt, Ghana: a new category of ore forming fluids? Mineral. Depos., 33: $317-319$.

Kolb J., Kisters A.F.M., Hoernes S., Meyer F.M. 2000. The origin of fluids and nature of fluid-rock interaction in mid-crustal auriferous mylonites of the Renco mine, southern Zimbabwe. Mineral. Depos., 35: 109-125.

Lamarão C.N., Dall'Agnol R., Lafon, J.M. 2001. Geocronologia e geoquímica das associações vulcânicas e plutônicas da região de Vila Riozinho, Província Aurífera do Tapajós. In: SBG-NO, Simp. Geol. Amazônia, 7, Resumos expandidos (CD-ROM).

Nunes C.M.D., Juliani C., Corrêa-Silva R.H., Monteiro L.V.S., Bettencourt J.S., Neumann R., Alcover Neto A., Rye R.O. 2001. Caracterização de um sistema epitermal high-sulfidation vulcânico paleoproterozóico da Província Aurífera do Tapajós, Pará. In: SBGNO, Simp. Geol. Amazônia, 7, Resumos expandidos (CD-ROM).

Ricci P.S.F., Vasquez M.L, Santos A., Klein E.L., Jorge-João X.S., Martins R.C. 1999. Suíte Intrusiva Creporizão - Província Tapajós: proposta e critérios de definição. In: SBG-NO, Simp. Geol. Amazônia, 6, Boletim de resumos expandidos, 519-522.

Ramboz C., Pichavant M., Weisbrod A. 1982. Fluid immiscibility in natural processes: Use and misuse of fluid inclusion data. II. Interpretation of fluid inclusion data in terms of immiscibility. Chem. Geol., 37: 29-48.

Robert F. \& Kelly W.C. 1987. Ore-forming fluids in Archean gold-bearing quartz veins at the Sigma Mine, Abitibi greenstone belt, Quebec, Canada. Econ. Geol., 82, 1464-1482.

Robert F. \& Poulsen K.H. 2001. Vein formation and deformation in greenstone gold deposits. In Richards, J.P. \& Tosdal, R.M. (eds.) Structural controls on ore genesis, Reviews in Economic Geology, 14: 111-155

Roedder E. 1984. Fluid Inclusions. Mineralogical Society of America, Reviews in Mineralogy, 12, $644 \mathrm{p}$.

Rosa-Costa L.T. \& Carvalho J.M.A. 1998. Estudo das mineralizações auríferas do Tapajós - áreas Rio Novo e Marupá. Belém. CPRM (relatório inédito), $46 \mathrm{p}$.

Rosa-Costa L.T. \& Carvalho, J.M.A. 1999. Tipologia de mineralizações auríferas da região sul da Província Tapajós - Pará. In: SBG-NO, Simpósio de Geologia da Amazônia, 6, Boletim de resumos expandidos, 176-179.

Santos. R.A. 1999. Controle estrutural das mineralizações de ouro da Província Mineral do Tapajós. Salvador, CPRM (relatório inédito).

Santos R.A. 2000. Formation of gold-quartz veins controlled by the strike-slip fault mega-system in Tapajós Mineral Province, Amazon, Brazil. In: International Geological Congress, 31, Rio de Janeiro, abstracts (CD-ROM).

Santos J.O.S., Hartmann L.A., Gaudette H.E., Groves D.I., McNaughton N.J., Fletcher I.R. 2000. A new understanding of the provinces of the Amazon Craton based on integration of field mapping and $U$ $\mathrm{Pb}$ and $\mathrm{Sm}-\mathrm{Nd}$ geochronology. Gondwana Research, 3: 453-488.

Santos J.O.S., Groves D.I., Hartmann L.A., Moura M.A., McNaughton N.J. 2001. Gold deposits of the Tapajós and Alta Floresta Domains, Tapajós-Parima orogenic belt, Amazon Craton, Brazil. Mineral. Dep., 36: 279-299.

Schwartz M.O., Oberthür T., Amanor T., Gyapong W.A. 1992. Fluid inclusion re-equilibration ant P-T-X constraints on fluid evolution in the Ashanti gold deposit, Ghana. European J.Mineral., 4: 1017-1033.

Skinner B. 1997. Hydrothermal mineral deposits: what we do and don't know. In: Barnes H.L. (ed) Geochemistry of hydrothermal ore deposits. New York, John Wiley \& Sons, Inc., pp.: 1-29.

Vasquez M.L., Klein E.L., Quadros M.L.E.S., Bahia R.B.C., Santos A., Ricci P.S.F., Sachett C.R., Silva C.M.G., Macambira M.J.B. 1999. Magmatismo Uatumã na Província Tapajós - novos dados geocronológicos. In: SBG-NO, Simp. Geol. Amazônia, 6, Boletim de resumos expandidos, 471-474.

Vasquez M.L., Ricci P.S.F., Klein E.L. 2002. Granitóides pós-colisionais da porção leste da Província Tapajós. In: Klein E.L., Vasquez M.L. Rosa-Costa L.T. (eds.) Contrib. Geol. Amazônia, Belém, SBGNO, v.3, 67-83.

Vearncombe J.R. 1993. Quartz vein morphology and implications for formation depth and classification of Archaean gold-vein deposits. Ore Geol. Reviews, 8: 407-424.

Wilkinson J.J. 2001. Fluid inclusions in hydrothermal ore deposits. Lithos, 55: $229-272$.

Manuscrito A-1383

Recebido em 15 de outubro de 2002

Revisão dos autores em 20 de novembro de 2003 Revisão aceita em 25 de novembro de 2003 